\title{
Hepatic Cholesterol-25-Hydroxylase Overexpression Improves Systemic Insulin Sensitivity in Mice
}

\author{
Britta Noebauer, ${ }^{1}$ Alexander Jais, ${ }^{1}$ Jelena Todoric, ${ }^{2}$ Klaus Gossens, ${ }^{3}$ \\ Hedwig Sutterlüty-Fall, ${ }^{4}$ and Elisa Einwallner ${ }^{1}$ \\ ${ }^{1}$ Department of Laboratory Medicine, Medical University of Vienna, Vienna, Austria \\ ${ }^{2}$ Laboratory of Gene Regulation and Signal Transduction, Departments of Pharmacology and Pathology, \\ UCSD School of Medicine, San Diego, CA, USA \\ ${ }^{3}$ Max Planck Institute for Immunobiology and Epigenetics, Freiburg, Germany \\ ${ }^{4}$ Institute of Cancer Research, Department of Medicine I, Comprehensive Cancer Center, Medical University of Vienna, Vienna, Austria
}

Correspondence should be addressed to Elisa Einwallner; elisa.einwallner@meduniwien.ac.at

Received 13 November 2016; Revised 4 January 2017; Accepted 31 January 2017; Published 19 February 2017

Academic Editor: Toshiyasu Sasaoka

Copyright (C) 2017 Britta Noebauer et al. This is an open access article distributed under the Creative Commons Attribution License, which permits unrestricted use, distribution, and reproduction in any medium, provided the original work is properly cited.

Obesity is a major risk factor for several diseases including diabetes, heart disease, and some forms of cancer and due to its rapidly increasing prevalence it has become one of the biggest problems medicine is facing today. All the more surprising, a substantial percentage of obese patients are metabolically healthy when classified based on insulin resistance and systemic inflammation. Oxysterols are naturally occurring molecules that play important role in various metabolic and inflammatory processes and their levels are elevated in patients suffering from obesity and diabetes. 25-Hydroxycholesterol (25-OHC) is produced in cells from cholesterol by the enzyme cholesterol 25 -hydroxylase $(\mathrm{Ch} 25 \mathrm{~h})$ and is involved in lipid metabolism, inflammatory processes, and cell proliferation. Here, we investigated the role of hepatic $\mathrm{Ch} 25 \mathrm{~h}$ in the transition from metabolically healthy obesity to insulin resistance and diabetes. Using several different experimental approaches, we demonstrated the significance of Ch25h on the border of "healthy" and "diseased" states of obesity. Adenovirus-mediated Ch25h overexpression in mice improved glucose tolerance and insulin sensitivity and lowered HOMA-IR. Our data suggest that low hepatic Ch25h levels could be considered a risk marker for unhealthy obesity.

\section{Introduction}

Obesity has become a major global health burden due to rapid increase in prevalence that has more than doubled since 1980 (WHO, 2014). There are over 1.9 billion overweight adults worldwide and more than 600 million of them are classified as obese. Obesity has been officially recognized as a disease by American Medical Association and it poses one of the main risk factors for chronic diseases such as type 2 diabetes, heart disease, hypertension, osteoarthritis, sleep apnea, and several types of cancer $[1,2]$. In addition, obesity is a part of metabolic syndrome, which is a group of linked disorders including elevated blood pressure, fasting plasma glucose, and serum triglycerides and low high-density lipoprotein (HDL) levels, associated with increased risk of heart disease and type 2 diabetes [3]. Interestingly, up to one out of four obese individuals is metabolically healthy [4] classified on the basis of indices for insulin resistance and systemic inflammation.

Factors discriminating between the "healthy" and the "unhealthy" obese individuals are still ill-defined. Metabolically healthy obesity is associated with smaller adipocyte size and higher ratio of subcutaneous versus visceral fat depots [5]. The amount of visceral fat also correlates with intrahepatic triglyceride content [6-8]. In line with this, impaired insulin action is linked to accumulation of saturated fatty acids and ectopic lipids in the liver [9]. Increased hepatic lipogenesis is one of the hallmarks of many obesity 
and diabetes models [10] and hepatic steatosis might be a major mechanism for developing insulin resistance [1113]. The relationship between lipid accumulation in various tissues and insulin resistance still needs further studies; however, it is possible that ectopic triglycerides in liver are the primary cause for metabolic abnormalities that accompany "unhealthy" obesity.

Oxygenated derivatives of cholesterol, oxysterols, are elevated in patients suffering from obesity, diabetes, hypercholesterolemia, and atherosclerosis [14-16]. The central role of oxysterols is the regulation of cholesterol metabolism, which is controlled, in part, by their binding to nuclear receptors (NRs), such as liver X receptors (LXRs) and retinoidrelated orphan receptors RORs [17, 18]. The activation of LXRs induces a range of genes that are involved in cholesterol transport and excretion and encode anti-inflammatory proteins [19-21]. In general, when cells are challenged with excess cholesterol, LXR-dependent transcriptional signaling enables them to reconstitute cholesterol homeostasis [21]. In addition, LXRs have been shown to suppress immune responses mediated by macrophages and other immune cells $[22,23]$. However, at the same time LXRs are able to directly activate lipogenic genes and stimulate de novo lipogenesis $[20,24]$ which can result in hepatic steatosis and insulin resistance [25]. There are several naturally occurring oxysterols including the three most prominent ones, 25hydroxycholesterol (25-OHC), 24-hydroxycholesterol, and 27-hydroxycholesterol. 25-OHC has been shown to be a regulator of lipid metabolism, inflammatory processes, and cell proliferation [26]. Beyond its role in regulation of lipid metabolism 25-OHC can serve both pro- and antiinflammatory functions [27, 28]. The formation of $25-\mathrm{OHC}$ is catalyzed by the enzyme cholesterol 25-hydroxylase (Ch25h) $[29,30]$. Most tissues show very low Ch25h expression levels [31]; however in vivo treatment with Toll-like receptor (TLR) agonists increases expression, most notably in liver and macrophages that are a potent source of inducible $\mathrm{Ch} 25 \mathrm{~h}$ $[32,33]$. Here we show that $\mathrm{Ch} 25 \mathrm{~h}$ levels are decreased in obese insulin resistant compared to obese insulin sensitive mice and an increase in hepatic Ch25h mRNA expression and protein levels improves insulin sensitivity, which makes it an important factor in the transition from "healthy" to "diseased" obesity.

\section{Materials and Methods}

2.1. Reagents. All chemicals and reagents were obtained from Sigma, unless stated otherwise.

2.2. $q P C R$. Quantitative PCR analysis was conducted as previously described [34]. Total RNA was extracted from tissues and cells using RNA isolation kits (RNeasy, QIAGEN; TRIzol, Invitrogen). The isolated total RNA was converted into cDNA via reverse transcription using commercially available kits (High-Capacity cDNA Reverse Transcription Kit, Applied Biosystems). iQ SYBR Green Supermix (BioRad Laboratories) was used for qPCR reactions. Primeronly controls were included for ensuring the absence of primer dimers and a check for unspecific products was conducted by performing a postamplification melting curve analysis. Normalization was done by normalizing threshold cycles (Ct values) to acidic ribosomal phosphoprotein P0 (Rplp0) within each sample for obtaining $\Delta \mathrm{Ct}$ values samplespecific (= Ct gene of interest $-\mathrm{Ct}$ Rplp0). Fold expression levels were obtained by calculating $2^{-\Delta \Delta \mathrm{Ct}}$ levels $(\Delta \Delta \mathrm{Ct}=$ $\Delta \mathrm{Ct}$ treatment $-\Delta \mathrm{Ct}$ control).

2.3. Western Blot. Tissues were homogenized in RIPA buffer (0.5\% NP-40, $0.1 \%$ sodium deoxycholate, $150 \mathrm{mM} \mathrm{NaCl}$, and $50 \mathrm{mM}$ Tris- $\mathrm{HCl}, \mathrm{pH} 7.5)$ containing protease inhibitors (Complete Mini, Roche). Homogenate was cleared by centrifugation at $4^{\circ} \mathrm{C}$ for $30 \mathrm{~min}$ at $15,000 \times \mathrm{g}$, following the recovery of the supernatant containing the protein fraction. The BCA Protein Assay Kit (Pierce) was used for determination of protein concentration in the supernatant. SDS-PAGE resolving of $20 \mu \mathrm{g}$ of protein was followed by transfer to PVDF membranes (GE Healthcare). 5\% BSA in Tris-buffered saline containing $0.2 \%$ Tween- 20 (TBS-T) was used for blocking membranes, which were then incubated with primary antibodies at $4^{\circ} \mathrm{C}$ overnight. The following antibodies were used: anti- $\beta$-actin (1:500; A5441, Sigma) and anti-CH25H antibody (1:1000 ab76478, Abcam). Incubated membranes were washed and probed with appropriate secondary antibody (NA 934, anti-rabbit IgG, 1:20,000; NA 931, anti-mouse IgG, 1:20,000; GE Healthcare). Detection of antigen-specific binding of antibodies was performed with SuperSignal West Femto and Pico Kits (Pierce) by the use of a ChemiDoc XRS Imager (Bio-Rad). Image Lab Software version 3.0.1 was used for Image analysis (Bio-Rad).

2.4. 25-Hydroxycholesterol ELISA. Liver samples were homogenized and run along with standards supplied with the kit as per the user manual supplied with 25-hydroxycholesterol ELISA kit (\#B0344, Glory Science). Optical density (OD) was measured at $450 \mathrm{~nm}$.

2.5. Animals. Male C57BL/6J mice (wild type, wt) were purchased from the Charles River Laboratories (Sulzfeld, Germany). All mice were maintained on a 12-hour light/dark cycle and had free access to food and water. Mice were sacrificed by cervical dislocation and liver samples were collected and immediately snap-frozen in liquid nitrogen. All animal procedures used in this study were reviewed and approved by the Animal Care and Use Committee of the Medical University of Vienna and conducted according to FELASA guidelines. At 6 weeks of age, mice were placed for 24 weeks on high-fat (60\% fat calories, D12492, Research Diets Inc., New Brunswick, NJ, USA; $n=10$ /group) and low-fat (10\% fat calories, D12450B, Research Diets Inc.; $n=$ 10/group) diet to induce obesity and to serve as lean controls. Liver protein samples were collected from lean and obese C57BL/6J mice for further experiments.

2.6. Oral Glucose Tolerance Test. Following an overnight fast, mice were given glucose $(1 \mathrm{~g} / \mathrm{kg})$ via oral gavage. At the indicated time points, blood samples for determination of 


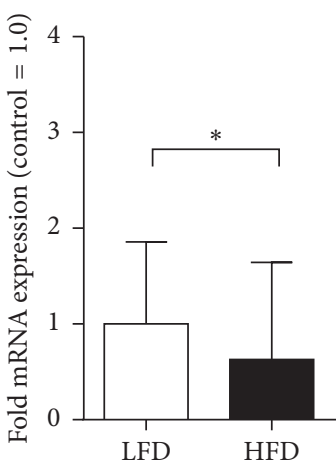

(a)

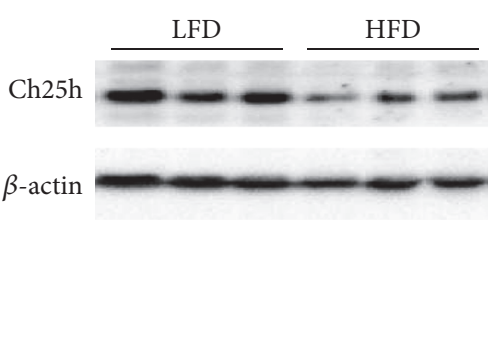

(b)

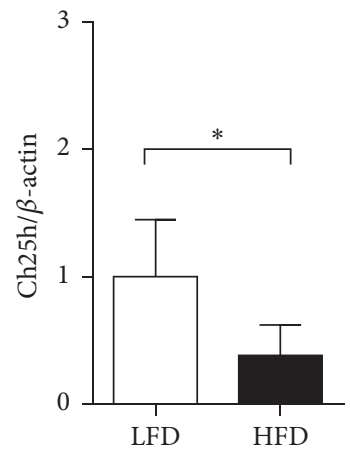

FIGURE 1: HFD treatment on mice resulted in decreased hepatic Ch25h protein levels. (a) Ch25h mRNA levels in isolated livers obtained from mice on HFD and control LFD. mRNA levels of hepatic Ch25h levels in LFD mice were xxx-fold higher comparing to mice on HFD. ${ }^{*} p=0.036, n=10$ per group. (b) Immunoblot analysis of Ch25h protein in isolated livers obtained from mice on HFD and control LFD. Mice on HFD showed a clear reduction in liver Ch25h protein levels comparing to the littermate controls. $\beta$-Actin was used as a loading control. ${ }^{*} p=0.046, n=3$ per group.

glucose and insulin levels were collected from the tail vein. An Accu-Chek (Roche) glucometer was used for assessing glycemia. Levels of plasma insulin were determined by the use of Ultrasensitive Mouse Insulin ELISA kit (Mercodia).

2.7. Insulin Tolerance Test. Following a 2-hour fast, insulin tolerance was assessed by the intraperitoneal administration of regular human insulin (0.75 U/kg Actrapid; Novo Nordisk) and monitoring blood glucose. Hepatic insulin sensitivity was assessed in adenovirus-injected mice by performing low-dose insulin tolerance tests $(0.1 \mathrm{U} / \mathrm{kg})$.

2.8. Obese Insulin Sensitive (obIS) and Obese Insulin Resistant (obIR) C57BL/6J Mice. As previously described [35], we generated a large cohort of high-fat diet- (HFD-) treated male C57BL/6J mice and stratified them according to glucose and insulin tolerance. Subgroups were selected by exhibiting either insulin sensitivity (IS, $n=5$ /group) or insulin resistance (IR, $n=5$ /group) despite comparable weight gain on the HFD. Liver samples were collected from these obese insulin sensitive (obIS) and obese insulin resistant (obIR) C57BL/6J mice fed for further analyses.

2.9. Adenovirus Experiments. Adenovirus experiments targeting livers were conducted with in vivo grade $\mathrm{CH} 25 \mathrm{H}$ (AdCH25H) and LacZ (AdLacZ) expressing viruses, produced according to published protocols [36]. FuGENE 6 Transfection Reagent (Roche) was used for cotransfecting Sfi I-digested Adlox plasmid DNA with psi5 DNA into Cre8 cells. Cells were collected by centrifugation, three days after transfection, and the recombined viruses were extracted from cell pellets by four cycles of freeze and thaw. Centrifugation was used to remove cell debris. When selecting for the recombined adenoviruses, we performed seven reinfection cycles in Cre8 cells. Adenoviral particles were amplified by the use of HEK293 cells (ATCC). Purification of amplified AdCH25H and AdLacZ was achieved by chloride density-gradient ultracentrifugation. They were then collected from the gradient, diluted in $2 \mathrm{x}$ storage buffer $1: 1$ (10 mM Tris $\mathrm{pH} 8.0,100 \mathrm{mM} \mathrm{NaCl}, 0.1 \%$ BSA, and $50 \%$ glycerol), and afterwards stored at $-20^{\circ} \mathrm{C}$ in small aliquots. The in vitro tail vein injections were implemented as previously described [37]. As recommended, a total volume of $200 \mu \mathrm{l}$ of AdHO-1 and AdLacZ particles diluted with PBS $\left(0.2 \times 10^{9} \mathrm{pfu} / \mathrm{g}\right.$ bodyweight $)$ was injected into the tail vein of the respective recipient mice. After day 5 and day 7 of postinjection, OGTTs and ITTs were performed.

\section{Results}

3.1. Liver Ch25h Expression Is Decreased during HFD. It has been shown that $\mathrm{Ch} 25 \mathrm{~h}$ is involved in the regulation of lipid metabolism; therefore our first aim was to investigate the role of hepatic Ch25h levels in obesity. We challenged C57BL/6J mice with high-fat diet (HFD) and observed a clear reduction of Ch25h mRNA and protein levels compared to controls in the isolated livers of mice on HFD treatment (Figures 1(a) and 1(b)). Recently, contrary to our data, GuillemotLegris and colleagues indicated that the levels of hepatic 25hydroxycholesterol were not affected during a HFD treatment in mice [38]. This led us to the search for published human datasets on $\mathrm{CH} 25 \mathrm{H}$ levels in obesity.

3.2. $\mathrm{CH} 25 \mathrm{H}$ Levels Decrease in Livers of Obese Subjects but Not in Livers of Diabetic Patients. We performed an unbiased search for human datasets and identified one human liver dataset (NCBI, GEO dataset GDS3876), comparing lean and obese patients with and without type 2 diabetes. In humans again, $\mathrm{CH} 25 \mathrm{H}$ levels in liver samples are consistently lower in obese patients. The data shows a tendency of lower $\mathrm{CH} 25 \mathrm{H}$ levels between only obese and obese patients with type 2 diabetes poorly controlled whereas this effect was lost in obese patients with type 2 diabetes mellitus when well 


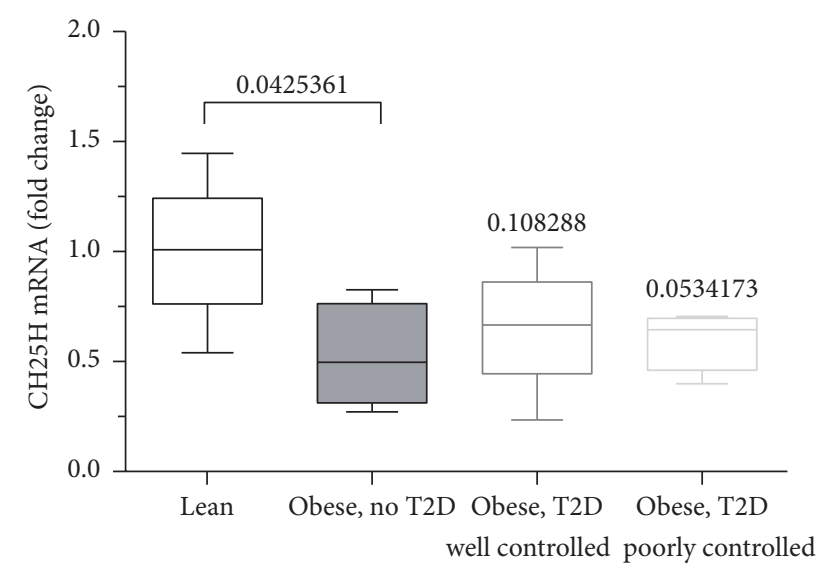

FIgURE 2: Dataset on liver $\mathrm{CH} 25 \mathrm{H}$ mRNA levels in lean subjects and obese patients with and without type 2 diabetes. $\mathrm{CH} 25 \mathrm{H}$ mRNA levels are decreased in obese patients without diabetes versus lean subjects. Levels of obese patients showing poorly controlled type 2 diabetes patients are decreased as well whereas $p$ values for mRNA levels of obese, well controlled type 2 diabetes patients are not significant. GDS3876/206932_at/CH25H. Obese patients with and without type 2 diabetes: liver. Homo sapiens.

controlled (Figure 2). This led us to further investigate the role of hepatic $\mathrm{CH} 25 \mathrm{H}$ levels in insulin sensitivity.

\subsection{Ch25h Protein Levels Are Decreased in Insulin Resistant} Mice. Exploring the link between Ch25h and "healthy" versus "diseased" states of obesity, we measured hepatic Ch25h levels in two cohorts of male C57BL/6J mice that were fed HFD and stratified according to their response to oral glucose and insulin tolerance tests [35].

Interestingly, our results showed a notable decrease in the levels of hepatic Ch25h mRNA of the mice belonging to the obese insulin resistant subpopulation (Figures 3(a) and 3(b)). Therefore, Ch25h shows significant predictive power in distinguishing these two groups and could be considered as a marker of metabolically diseased obesity. Since hepatic $\mathrm{Ch} 25 \mathrm{~h}$ was downregulated in mice treated with high-fat diet, in line with published human dataset as shown in Figure 2, in order to corroborate this finding we performed the overexpression of hepatic $\mathrm{Ch} 25 \mathrm{~h}$ via tail vein injection of adenoviral Ch25h under basal conditions (AdCh25h). Successful upregulation of Ch25h mRNA and protein levels is shown in Figures 4(a) and 4(b), respectively. Adeno-LacZ transduced mice served as control. To examine whether the upregulation of $\mathrm{Ch} 25 \mathrm{~h}$ protein directly influences the levels of 25-hydroxycholesterol (25-OHC) in hepatic tissue, we measured 25-OHC levels in liver biopsies of AdCh25h and AdLacZ treated mice (Figure 4(c)). 25-OHC levels were significantly elevated in the AdCh $25 \mathrm{~h}$ group compared to controls.

Importantly, Ch25h overexpression in normal chow-fed C57BL/6J mice further improved glucose tolerance, insulin sensitivity, and lowered HOMA-IR relative to Adeno-LacZ injected controls (Figures 5(a)-5(c)). This in vivo gain-offunction study indicates elevated hepatic Ch25h levels are able to improve insulin sensitivity and therefore induce a metabolically healthy form of obesity.

\section{Discussion}

The idea that oxysterols are general regulators of lipid metabolism is not new, since evidence exists for their activating or repressing interactions with NRs [18-20, 39]. 24SHydroxycholesterol (24-OHC) levels are involved in regulating cholesterol homeostasis in the brain [40] and were found to play a role in neurodegenerative diseases [41-46]. Another recent study by Guillemot-Legris and colleagues [38] observed not only a decrease of $4 \beta$-OHC in the plasma of human obese patients, but also the fact that the levels in the liver, hypothalamus, and adipose tissue were significantly lowered. Literature pointed out a metabolic role of 27and 25-hydroxycholesterol in the liver, the key regulator of cholesterol metabolism in an organism. Administration of $27-\mathrm{OHC}$ can reduce hepatic inflammation and regulate the intracellular distribution of cholesterol [47-49]. Inflammation is one of the key events that triggers insulin resistance and subsequently hyperglycemia in type 2 diabetes [50]. Reboldi and colleagues identified Ch25h as a crucial part in the negative feedback mechanism that regulates production of IL-1 family cytokines during inflammatory processes which involve type I IFN [51]. They also showed that 25$\mathrm{HC}$ has a repressive effect on Illb expression and is a broad inhibitor of inflammasome activity. When they tested the impact of Ch25h-deficiency on serum levels of inflammatory markers, they observed that $\mathrm{Ch} 25 \mathrm{~h}$-deficient mice showed higher levels of IL-1 $\alpha$, IL-1 $\beta$, and IL-18 upon LPStreatment, compared to controls [51]. This is in line with our observations that insulin resistant obese mice showed a significant decrease in Ch25h mRNA and protein levels in the liver, compared to their metabolically healthy obese littermates. Supporting these findings in the context of obesity and diabetes, Osborn and colleagues observed significantly reduced $\mathrm{HbAlc}$ levels when they treated mice suffering from T2D with anti-IL-1 $\beta$ antibodies, which indicates a beneficial effect on glucose tolerance and/or insulin resistance [52]. The role of inflammasomes in metabolic disorders, including obesity and diabetes, has been extensively studied over the last years. There are several reported inflammasomes, but one of the most prominent is the NLRP3 inflammasome, which has been shown to have a very broad range of targets [53]. NLRP3-deficient mice have been shown to have improved glucose tolerance and insulin sensitivity [54]. Similar to these findings, Wen and colleagues have studied the effects of saturated versus unsaturated fatty acids on the release of chemokines and the NLRP3 inflammasome activity. They could show that the saturated fatty acid, whose plasma concentration is clearly elevated after a HFD, has a trigger role in the NLRP3-dependent release of IL-1 $\beta$ and IL-18 and prevents regular insulin signaling in target tissues eventuating in insulin resistance, whereas administering unsaturated fatty acids had no influence on inflammasome activation or IL-1 $\beta$ release [55]. Collectively, literature indicates the activation of inflammasomes and 


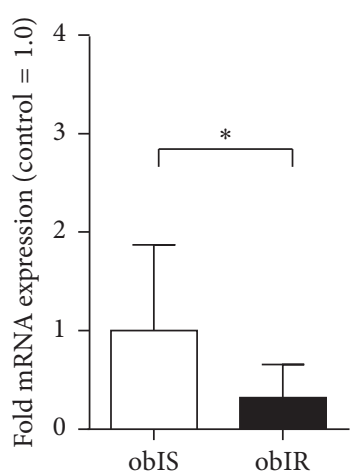

(a)

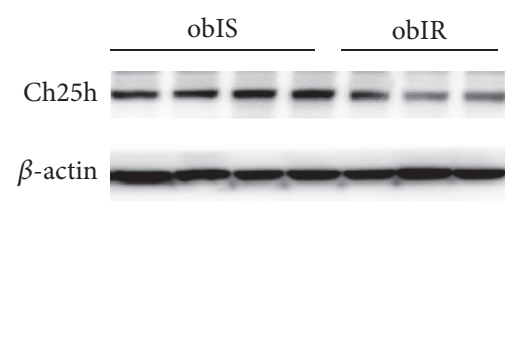

(b)

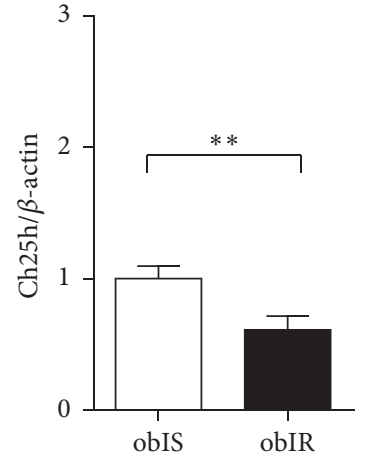

FIgURE 3: Obese IS mice show higher hepatic Ch25h levels than obese IR mice. (a) Relative Ch25h levels in isolated livers obtained from obese IR and obese IS C57BL/6J mice after 16 weeks on HFD. mRNA levels of hepatic Ch25h levels in obese IS mice were significantly higher comparing to obese IS mice. ${ }^{*} p=0.020, n=5$ per group. (b) Immunoblot analysis of Ch25h protein in isolated livers obtained from obese IR and obese IS C57BL/6J mice after 16 weeks on HFD. For immunoblot $\beta$-actin was used as a loading control. ${ }^{* *} p=0.002, n=3-4$ per group.

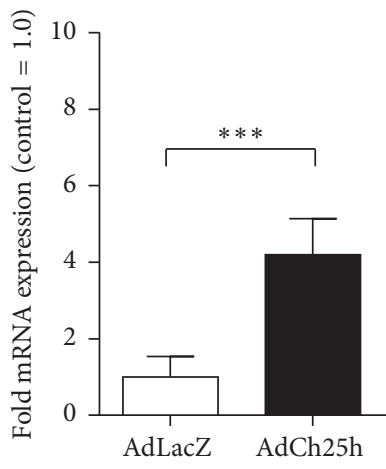

(a)

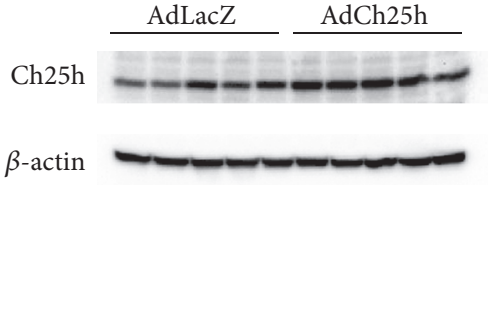

(b)

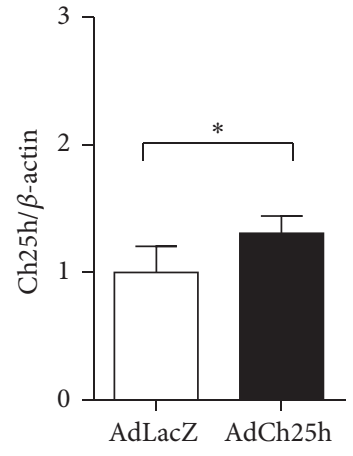

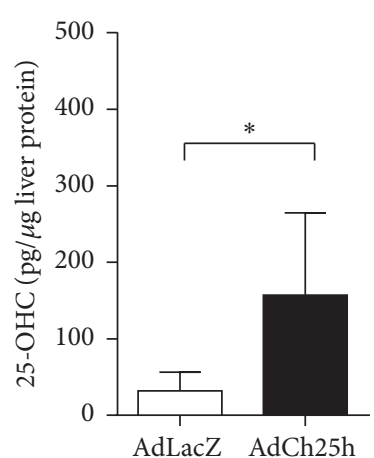

(c)

FIgURE 4: Tail vein injection of adenoviral Ch25h showed a successful hepatic Ch25h and 25-hydroxycholesterol overexpression. (a) Relative Ch25h levels in AdLacZ and AdCh25h transduced livers. mRNA levels of hepatic Ch25h levels increased 5-fold compared to AdLacZ injected controls. ${ }^{* * *} p<0.001, n=5$ per group. (b) Ch25h immunoblot of AdLacZ and AdCh25h transduced liver biopsies. Ch25h protein levels are clearly increased in livers of AdCh25h treated mice compared to AdLacZ transduced controls. $\beta$-Actin was used as a loading control. ${ }^{*} p=0.034, n=5$ per group. (c) 25-OHC levels in AdLacZ and AdCh25h transduced livers. 25-OHC levels are significantly increased in livers of AdCh25h treated mice compared to AdLacZ transduced controls. ${ }^{*} p=0.035, n=5$ per group.

release of IL- $1 \beta$ chemokines as pivotal underlying factors in the development of insulin resistance, which could be an explanation why the overexpression of Ch25h resulted in lower glucose and insulin levels and improved insulin sensitivity.

On the other hand, data exist showing that 25-HC can amplify secretion of inflammatory cytokines such as macrophage colony-stimulating factor (M-CSF), IL-6, and IL-8 $[56,57]$. These at first sight contradicting results indicate that 25 -HC cannot only be seen as a positive or negative regulator of the immune system. Wunderlich and colleagues have shown that IL-6 action, which originates from the activation of Kupffer cells in the liver, leads to a restricted inflammation, not only locally but also systemically, and therefore prevents insulin resistance [58]. Therefore, previously published data on 25-HC having an amplifying effect on the secretion of IL- 6 are in line with our results showing that overexpression of $\mathrm{Ch} 25 \mathrm{~h}$ was beneficial for further improving glucose tolerance, insulin sensitivity, and high HOMA-IR levels. As these different aspects of inflammatory processes in the development of insulin resistance and their correlation with elevated hepatic levels of Ch25h in mice could be explained with both attempts, based on already published data, further investigation is needed for elucidating the direct mechanisms and pathways responsible for our observed effects.

Recently, Tuong and colleagues reported that a siRNA mediated knock-down targeting $\mathrm{Ch} 25 \mathrm{~h}$ resulted in a significant increase in lipid droplets (LDs) in bone marrowderived macrophages (BMMs) [59]. Disorders of LDs, the major storage place for cholesterol, are associated with abnormalities in lipid trafficking that are closely associated with obesity, increased inflammatory responses, and insulin resistance. Converting cholesterol in hydroxycholesterol, or 

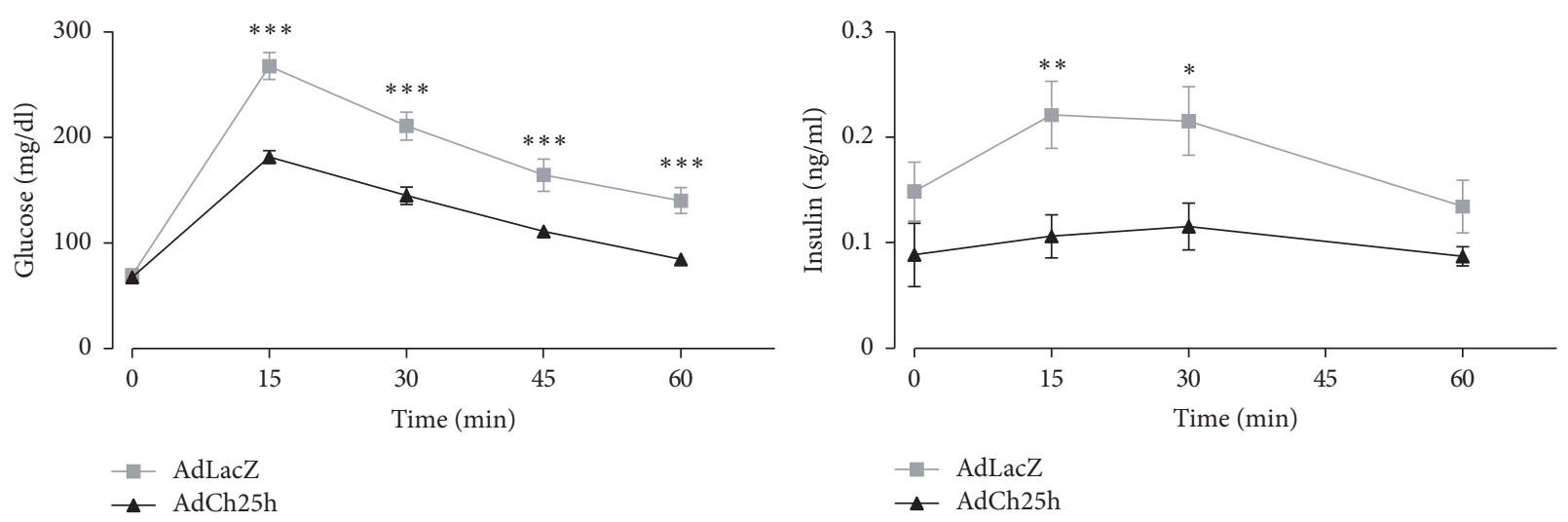

(a)

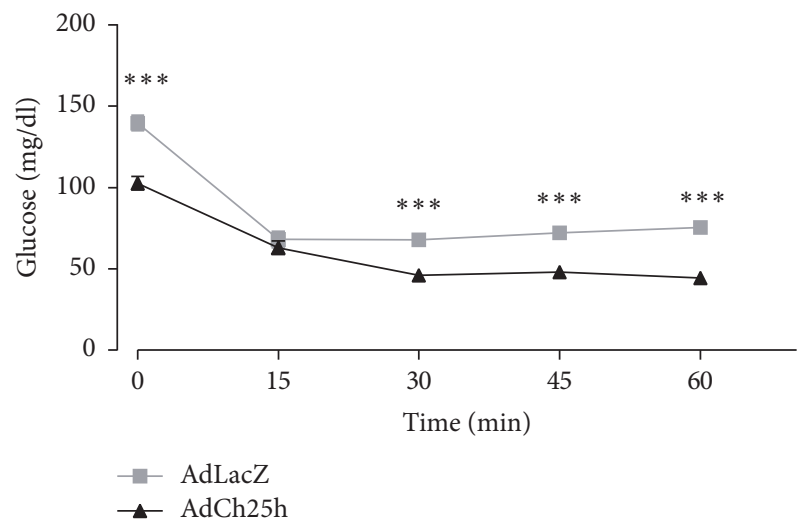

(b)

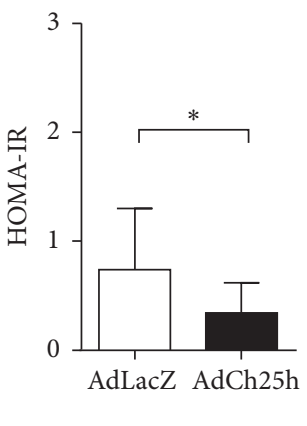

(c)

FIGURE 5: Overexpression of hepatic Ch25h improved glucose tolerance, insulin sensitivity, and lowered HOMA-IR. (a) Oral glucose tolerance tests (OGTT) performed in AdLacZ and AdCh25h transduced C57BL/6J animals. The glucose (mg/dl) and insulin (ng/ml) levels were measured 15, 30, 45, and $60 \mathrm{~min}$ after administering glucose solution $(1 \mathrm{~g} / \mathrm{kg})$. Blood glucose and insulin levels were significantly lower in the AdCh25h transduced subpopulation. Two-sided $p$ values obtained from unpaired $t$-test. Results are mean \pm SEM. ${ }^{*} p<0.05$, ${ }^{* *} p<0.01$, and ${ }^{* * *} p<0.001 . n=5$ per group. (b) Insulin tolerance tests (ITTs) on AdCh25h and AdLacZ transduced C57BL/6J mice. Regular human insulin was administered intraperitoneally and blood glucose levels were monitored and measured 15, 30, 45, and 60 min after injections. The blood glucose levels were clearly decreased in mice treated with AdCh25h. Two-sided $p$ values obtained from unpaired $t$-test. Results are mean \pm SEM. ${ }^{* * *} p<0.001, n=5$ per group. (c) HOMA-IR levels of AdLacZ and AdCh25h transduced C57BL/6J mice. Comparison of the control group to the AdCh25h treated mice showed a significant decrease in HOMA-IR levels. Results are mean \pm SEM. ${ }^{*} p<0.05, n=5$ per group.

in this case 25-HC, might be one of the mechanisms that cells use to rebalance excess amounts of cholesterol. This could explain why we observed low hepatic Ch25h levels in both insulin sensitive and insulin resistant obese mice.

To our knowledge, no reports are available on precise comparisons of Ch25h expression levels in the livers of obese insulin sensitive and insulin resistant subjects or mice. Our findings redefine the current view of this undervalued cholesterol hydroxylase enzyme and indicate a potent role of $\mathrm{Ch} 25 \mathrm{~h}$ in the regulation of the whole-body metabolism. Our data are also indicative of $\mathrm{Ch} 25 \mathrm{~h}$ expression where not only is it downregulated in the wake of obesity, but also its importance is mainly at the point of decision between insulin resistance and insulin sensitivity. Further studies are necessary regarding the exact mechanisms of hepatic Ch25h action in the development of insulin resistance and subsequently type 2 diabetes.

\section{Competing Interests}

The authors declare no conflict of interests associated with this manuscript.

\section{References}

[1] Managing Overweight and Obesity in Adults, Systematic Evidence Review from the Obesity Expert Panel 2013, http://www .nhlbi.nih.gov/sites/www.nhlbi.nih.gov/files/obesity-evidencereview.pdf.

[2] "Clinical Guidelines on the Identification, Evaluation and Treatment of Overweight and Obesity in Adults," http://www.nhlbi .nih.gov/files/docs/guidelines/ob_gdlns.pdf.

[3] J. M. Olefsky and C. K. Glass, "Macrophages, inflammation, and insulin resistance," Annual Review of Physiology, vol. 72, pp. 219246, 2010. 
[4] N. Stefan, H.-U. Häring, F. B. Hu, and M. B. Schulze, "Metabolically healthy obesity: epidemiology, mechanisms, and clinical implications," The Lancet Diabetes \& Endocrinology, vol. 1, no. 2, pp. 152-162, 2013.

[5] T. Tchkonia, T. Thomou, Y. Zhu et al., "Mechanisms and metabolic implications of regional differences among fat depots," Cell Metabolism, vol. 17, no. 5, pp. 644-656, 2013.

[6] J.-H. Hwang, D. T. Stein, N. Barzilai et al., "Increased intrahepatic triglyceride is associated with peripheral insulin resistance: in vivo MR imaging and spectroscopy studies," American Journal of Physiology-ndocrinology and Metabolism, vol. 293, no. 6, pp. E1663-E1669, 2007.

[7] N. Stefan, K. Kantartzis, and H.-U. Häring, "Causes and metabolic consequences of fatty liver," Endocrine Reviews, vol. 29, no. 7, pp. 939-960, 2008.

[8] B. L. Wajchenberg, "Subcutaneous and visceral adipose tissue: their relation to the metabolic syndrome," Endocrine Reviews, vol. 21, no. 6, pp. 697-738, 2000.

[9] V. T. Samuel and G. I. Shulman, "Mechanisms for insulin resistance: common threads and missing links," Cell, vol. 148, no. 5, pp. 852-871, 2012.

[10] R. V. Farese Jr., R. Zechner, C. B. Newgard, and T. C. Walther, "The problem of establishing relationships between hepatic steatosis and hepatic insulin resistance," Cell Metabolism, vol. 15 , no. 5 , pp. $570-573,2012$.

[11] K. F. Petersen and G. I. Shulman, "Cellular mechanism of insulin resistance in skeletal muscle," Journal of the Royal Society of Medicine, Supplement, vol. 95, no. 42, pp. 8-13, 2002.

[12] G. I. Shulman, "Cellular mechanisms of insulin resistance," The Journal of Clinical Investigation, vol. 106, no. 2, pp. 171-176, 2000.

[13] E. Fabbrini, F. Magkos, B. S. Mohammed et al., "Intrahepatic fat, not visceral fat, is linked with metabolic complications of obesity," Proceedings of the National Academy of Sciences of the United States of America, vol. 106, no. 36, pp. 15430-15435, 2009.

[14] D. Alkazemi, G. Egeland, J. Vaya, S. Meltzer, and S. Kubow, "Oxysterol as a marker of atherogenic dyslipidemia in adolescence," Journal of Clinical Endocrinology and Metabolism, vol. 93, no. 11, pp. 4282-4289, 2008.

[15] V. M. Olkkonen and M. Lehto, "Oxysterols and oxysterol binding proteins: role in lipid metabolism and atherosclerosis," Annals of Medicine, vol. 36, no. 8, pp. 562-572, 2004.

[16] B. Ziedén, A. Kaminskas, M. Kristenson et al., "Increased plasma $7 \beta$-hydroxycholesterol concentrations in a population with a high risk for cardiovascular disease," Arteriosclerosis, Thrombosis, and Vascular Biology, vol. 19, no. 4, pp. 967-971, 1999.

[17] T. Jakobsson, E. Treuter, J.-Å. Gustafsson, and K. R. Steffensen, "Liver X receptor biology and pharmacology: new pathways, challenges and opportunities," Trends in Pharmacological Sciences, vol. 33, no. 7, pp. 394-404, 2012.

[18] D. Töröcsik, A. Szanto, and L. Nagy, “Oxysterol signaling links cholesterol metabolism and inflammation via the liver X receptor in macrophages," Molecular Aspects of Medicine, vol. 30, no. 3, pp. 134-152, 2009.

[19] D. J. Peet, B. A. Janowski, and D. J. Mangelsdorf, “The LXRs: a new class of oxysterol receptors," Current Opinion in Genetics and Development, vol. 8, no. 5, pp. 571-575, 1998.

[20] J. R. Schultz, H. Tu, A. Luk et al., "Role of LXRs in control of lipogenesis," Genes and Development, vol. 14, no. 22, pp. 28312838, 2000.
[21] P. Tontonoz and D. J. Mangelsdorf, "Liver X receptor signaling pathways in cardiovascular disease," Molecular Endocrinology, vol. 17, no. 6, pp. 985-993, 2003.

[22] C. Traversari and V. Russo, "Control of the immune system by oxysterols and cancer development," Current Opinion in Pharmacology, vol. 12, no. 6, pp. 729-735, 2012.

[23] N. J. Spann and C. K. Glass, "Sterols and oxysterols in immune cell function," Nature Immunology, vol. 14, no. 9, pp. 893-900, 2013.

[24] J. J. Repa, G. Liang, J. Ou et al., "Regulation of mouse sterol regulatory element-binding protein-1c gene (SREBP-1c) by oxysterol receptors, $\operatorname{LXR} \alpha$ and $\operatorname{LXR} \beta$," Genes \& Development, vol. 14, no. 22, pp. 2819-2830, 2000.

[25] A. Grefhorst and E. J. Parks, "Reduced insulin-mediated inhibition of VLDL secretion upon pharmacologic alactivation of the liver X receptor in mice," Journal of Lipid Research, vol. 50, no. 7, pp. 1374-1383, 2009.

[26] S. Ren and Y. Ning, "Sulfation of 25-hydroxycholesterol regulates lipid metabolism, inflammatory responses, and cell proliferation," American Journal of Physiology-Endocrinology and Metabolism, vol. 306, no. 2, pp. E123-E130, 2014.

[27] T. Rosklint, B. G. Ohlsson, O. Wiklund, K. Norén, and L. M. Hultén, "Oxysterols induce interleukin- $1 \beta$ production in human macrophages," European Journal of Clinical Investigation, vol. 32, no. 1, pp. 35-42, 2002.

[28] M. C. O. Englund, A.-L. K. Karlsson, O. Wiklund, G. Bondjers, and B. G. Ohlsson, "25-Hydroxycholesterol induces lipopolysaccharide-tolerance and decreases a lipopolysaccharideinduced TNF- $\alpha$ secretion in macrophages," Atherosclerosis, vol. 158, no. 1, pp. 61-71, 2001.

[29] D. W. Russell, "Oxysterol biosynthetic enzymes," Biochimica et Biophysica Acta, vol. 1529, no. 1-3, pp. 126-135, 2000.

[30] R. S. Holmes, J. L. VandeBerg, and L. A. Cox, "Genomics and proteomics of vertebrate cholesterol ester lipase (LIPA) and cholesterol 25-hydroxylase (CH25H)," 3 Biotech, vol. 1, no. 2, pp. 99-109, 2011.

[31] E. G. Lund, T. A. Kerr, J. Sakai, W.-P. Li, and D. W. Russell, "cDNA cloning of mouse and human cholesterol 25-hydroxylases, polytopic membrane proteins that synthesize a potent oxysterol regulator of lipid metabolism," The Journal of Biological Chemistry, vol. 273, no. 51, pp. 34316-34327, 1998.

[32] U. Diczfalusy, K. E. Olofsson, A.-M. Carlsson et al., "Marked upregulation of cholesterol 25-hydroxylase expression by lipopolysaccharide," Journal of Lipid Research, vol. 50, no. 11, pp. 2258-2264, 2009.

[33] D. R. Bauman, A. D. Bitmansour, J. G. McDonald, B. M. Thompson, G. Liang, and D. W. Russell, "25-Hydroxycholesterol secreted by macrophages in response to Toll-like receptor activation suppresses immunoglobulin A production," Proceedings of the National Academy of Sciences of the United States of America, vol. 106, no. 39, pp. 16764-16769, 2009.

[34] J. Todoric, B. Strobl, A. Jais et al., "Cross-talk between interferon- $\gamma$ and hedgehog signaling regulates adipogenesis," Diabetes, vol. 60, no. 6, pp. 1668-1676, 2011.

[35] A. Jais, E. Einwallner, O. Sharif et al., "Heme oxygenase-1 drives metaflammation and insulin resistance in mouse and man," Cell, vol. 158, no. 1, pp. 25-40, 2014.

[36] S. Hardy, M. Kitamura, T. Harris-Stansil, Y. Dai, and M. L. Phipps, "Construction of adenovirus vectors through Cre-lox recombination," Journal of Virology, vol. 71, no. 3, pp. 1842-1849, 1997. 
[37] J. A. Pospisilik, C. Knauf, N. Joza et al., “Targeted deletion of AIF decreases mitochondrial oxidative phosphorylation and protects from obesity and diabetes," Cell, vol. 131, no. 3, pp. 476491, 2007.

[38] O. Guillemot-Legris, V. Mutemberezi, P. D. Cani, and G. G. Muccioli, "Obesity is associated with changes in oxysterol metabolism and levels in mice liver, hypothalamus, adipose tissue and plasma," Scientific Reports, vol. 6, Article ID 19694, 2016.

[39] W. Chen, G. Chen, D. L. Head, D. J. Mangelsdorf, and D. W. Russell, "Enzymatic reduction of oxysterols impairs LXR signaling in cultured cells and the livers of mice," Cell Metabolism, vol. 5, no. 1, pp. 73-79, 2007.

[40] C. Xie, E. G. Lund, S. D. Turley, D. W. Russell, and J. M. Dietschy, "Quantitation of two pathways for cholesterol excretion from the brain in normal mice and mice with neurodegeneration," Journal of Lipid Research, vol. 44, no. 9, pp. 1780-1789, 2003.

[41] A. Papassotiropoulos, D. Lütjohann, M. Bagli et al., "Plasma 24S-hydroxycholesterol: a peripheral indicator of neuronal degeneration and potential state marker for Alzheimer's disease," NeuroReport, vol. 11, no. 9, pp. 1959-1962, 2000.

[42] V. Leonia, T. Mastermanc, U. Diczfalusy, G. De Lucaa, J. Hillertc, and I. Björkhem, "Changes in human plasma levels of the brain specific oxysterol 24S-hydroxycholesterol during progression of multiple sclerosis," Neuroscience Letters, vol. 331, no. 3, pp. 163$166,2002$.

[43] H. Kölsch, R. Heun, A. Kerksiek, K. V. Bergmann, W. Maier, and D. Lütjohann, "Altered levels of plasma 24S- and 27hydroxycholesterol in demented patients," Neuroscience Letters, vol. 368, no. 3, pp. 303-308, 2004.

[44] L. A. Shobab, G.-Y. R. Hsiung, and H. H. Feldman, "Cholesterol in Alzheimer's disease," The Lancet Neurology, vol. 4, no. 12, pp. 841-852, 2005.

[45] A. Solomon, V. Leoni, M. Kivipelto et al., "Plasma levels of 24S-hydroxycholesterol reflect brain volumes in patients without objective cognitive impairment but not in those with Alzheimer's disease," Neuroscience Letters, vol. 462, no. 1, pp. 8993, 2009.

[46] V. Leoni, J. D. Long, J. A. Mills, S. Di Donato, and J. S. Paulsen, "Plasma 24S-hydroxycholesterol correlation with markers of Huntington disease progression," Neurobiology of Disease, vol. 55, pp. 37-43, 2013.

[47] J. G. Cyster, E. V. Dang, A. Reboldi, and T. Yi, "25-Hydroxycholesterols in innate and adaptive immunity," Nature Reviews Immunology, vol. 14, no. 11, pp. 731-743, 2014.

[48] U. Diczfalusy, "On the formation and possible biological role of 25-hydroxycholesterol," Biochimie, vol. 95, no. 3, pp. 455-460, 2013.

[49] V. Bieghs, T. Hendrikx, P. J. Van Gorp et al., "The cholesterol derivative 27-hydroxycholesterol reduces steatohepatitis in mice," Gastroenterology, vol. 144, no. 1, pp. 167.el-178.e1, 2013.

[50] M. Banerjee and M. Saxena, "Interleukin-1 (IL-1) family of cytokines: role in type 2 diabetes," Clinica Chimica Acta, vol. 413, no. 15-16, pp. 1163-1170, 2012.

[51] A. Reboldi, E. V. Dang, J. G. McDonald, G. Liang, D. W. Russell, and J. G. Cyster, "25-hydroxycholesterol suppresses interleukin1-driven inflammation downstream of type I interferon," Science, vol. 345, no. 6197, pp. 679-684, 2014.

[52] O. Osborn, S. E. Brownell, M. Sanchez-Alavez, D. Salomon, H. Gram, and T. Bartfai, "Treatment with an Interleukin 1 beta antibody improves glycemic control in diet-induced obesity," Cytokine, vol. 44, no. 1, pp. 141-148, 2008.
[53] A. M. K. Choi and K. Nakahira, "Dampening insulin signaling by an NLRP3 'meta-flammasome," Nature Immunology, vol. 12, no. 5, pp. 379-380, 2011.

[54] R. Zhou, A. Tardivel, B. Thorens, I. Choi, and J. Tschopp, "Thioredoxin-interacting protein links oxidative stress to inflammasome activation," Nature Immunology, vol. 11, no. 2, pp. 136-140, 2010.

[55] H. Wen, D. Gris, Y. Lei et al., "Fatty acid-induced NLRP3-ASC inflammasome activation interferes with insulin signaling," Nature Immunology, vol. 12, no. 5, pp. 408-415, 2011.

[56] E. S. Gold, A. H. Diercks, I. Podolsky et al., "25-Hydroxycholesterol acts as an amplifier of inflammatory signaling," Proceedings of the National Academy of Sciences of the United States of America, vol. 111, no. 29, pp. 10666-10671, 2014.

[57] A. Koarai, S. Yanagisawa, H. Sugiura et al., "25-Hydroxycholesterol enhances cytokine release and toll-like receptor 3 response in airway epithelial cells," Respiratory Research, vol. 13, article 63, 2012.

[58] F. T. Wunderlich, P. Ströhle, A. C. Könner et al., "Interleukin-6 signaling in liver-parenchymal cells suppresses hepatic inflammation and improves systemic insulin action," Cell Metabolism, vol. 12, no. 3, pp. 237-249, 2010.

[59] Z. K. Tuong, P. Lau, X. Du et al., "ROR $\alpha$ and 25-hydroxycholesterol crosstalk regulates lipid droplet homeostasis in macrophages," PLoS ONE, vol. 11, no. 1, Article ID e0147179, 2016. 


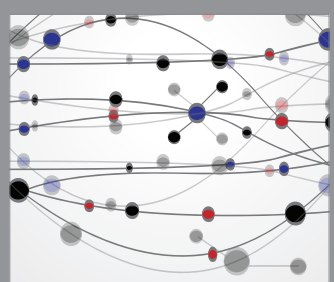

The Scientific World Journal
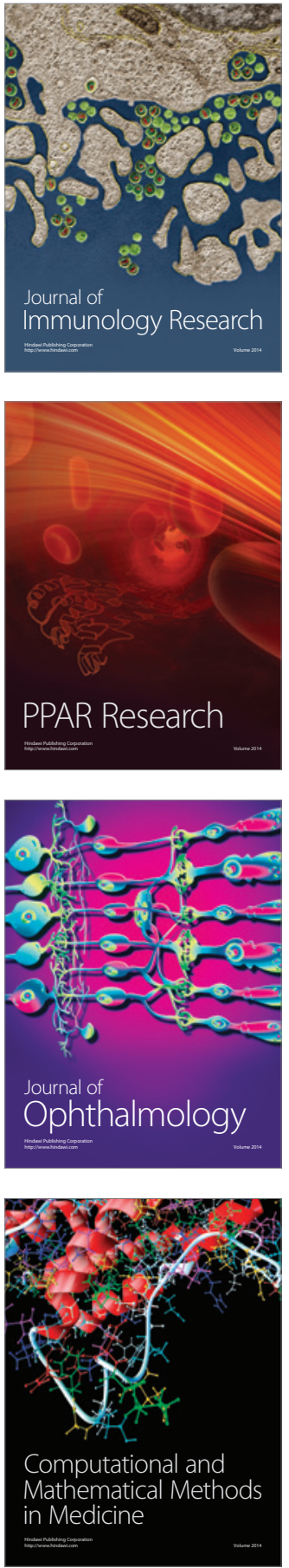

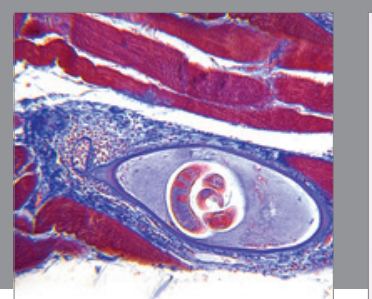

Gastroenterology Research and Practice
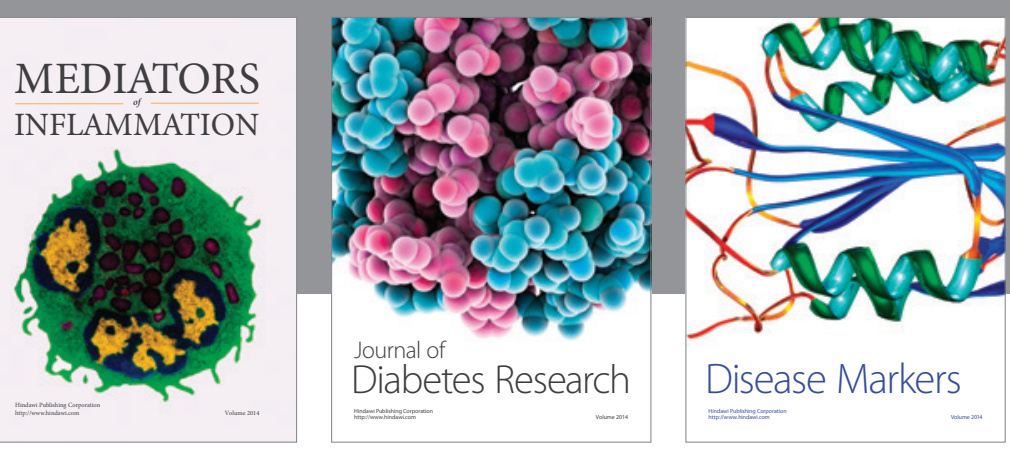

Disease Markers

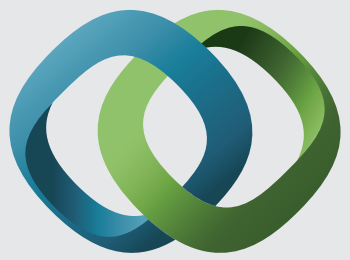

\section{Hindawi}

Submit your manuscripts at

https://www.hindawi.com
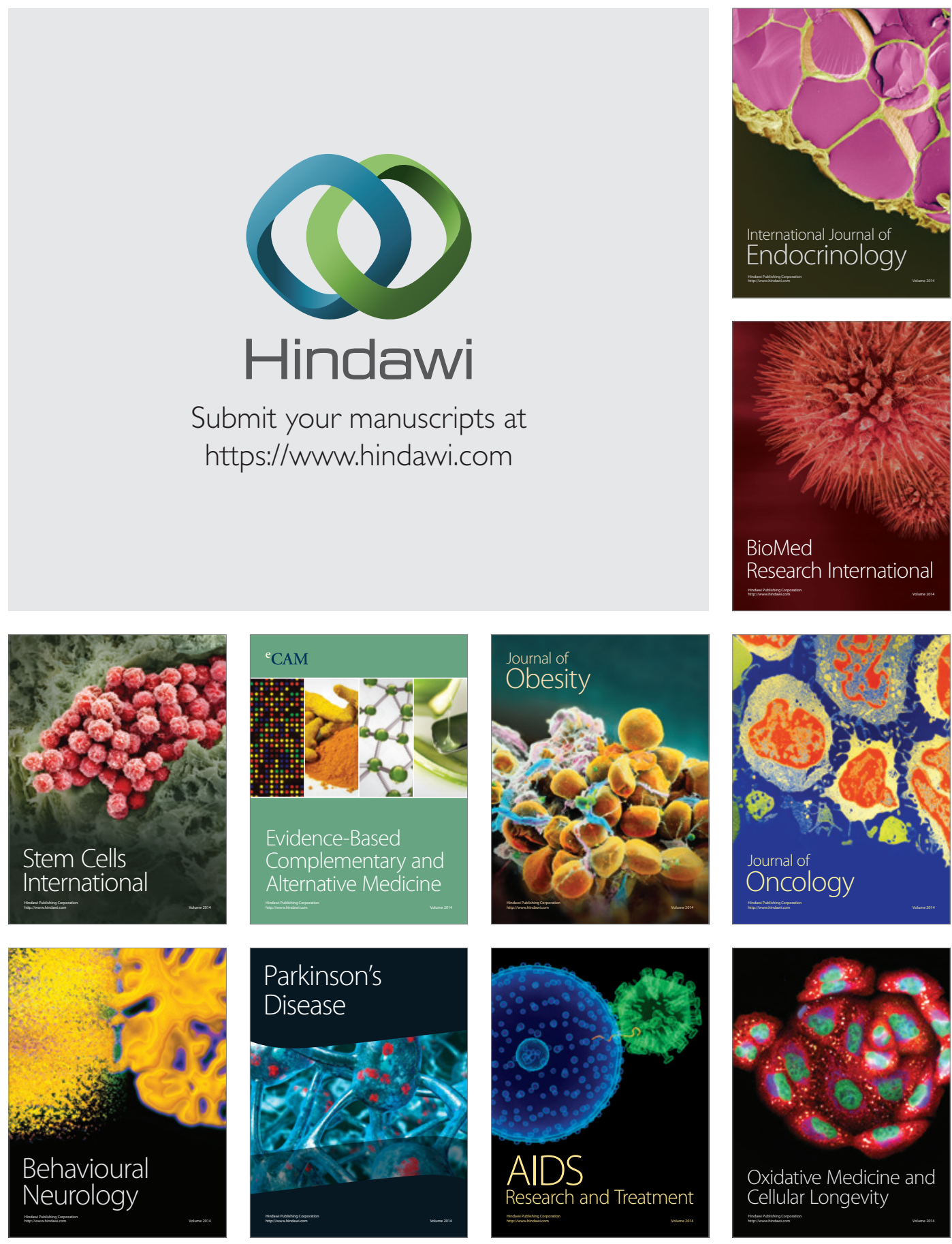